\title{
Ag/CuO/MCM-48 AS A POTENTIAL CATALYST FOR THE SYNTHESIS OF SYMMETRICAL AND UNSYMMETRICAL POLYHYDROQUINOLINES
}

\author{
NARGES BAZDID-VAHDATY ${ }^{a}$, MANOUCHEHR MAMAGHANI $^{b *}$, BEHZAD KHALILI $^{b}$ \\ AND FATEME TAVAKOLI ${ }^{b}$ \\ ${ }^{a}$ Department of Chemistry, University Campus 2, University of Guilan, P.O. Box 41335-1914 Rasht, Iran. \\ ${ }^{b}$ Department of Chemistry, Faculty of Science, University of Guilan, P.O. Box 41335-1914, Rasht, Iran.
}

\begin{abstract}
$\mathrm{Ag} / \mathrm{CuO} / \mathrm{MCM}-48$ as a heterogeneous catalyst was efficiently employed in the synthesis of diversely substituted symmetrical and unsymmetrical polyhydroquinoline by a multi-component reaction of arylaldehyde, dimedone, ethyl cyanoacetate and ammonium acetate. This novel method is simple, environmentally friendly, rapid, uses a recyclable catalyst and produces the products in high to excellent yields (83-97\%) and lower reaction times (17-35 min). The catalyst can be reused at least 10 times without any appreciable decrease in its catalytic activities.
\end{abstract}

Keywords: $\mathrm{Ag} / \mathrm{CuO} / \mathrm{MCM}-48$, Multi-components reaction, One-pot synthesis, Polyhydroquinoline.

\section{INTRODUCTION}

Polyhydroquinoline derivatives exhibit a variety of pharmacological properties such as vasodilator, bronchodilator, antiatherosclerotic, antitumor, geroprotective, hepatoprotective, HIV protease inhibition, selective antagonism of adenosine-A3 receptors, antidiabetic activities and the treatment of Alzheimer's disease [1-5]. Their 1,4-dihydropyridine unit was first recognized as calcium channel modulators but was later developed as cardiovascular and antihypertensive drugs including amlodipine, felodipine, nicardipine and nifedipine (Fig.1) [6-9].

Usually, polyhydroquinolines is accessed through Hantzsch reaction with several drawbacks such as low to moderate yields, besides requiring harsh conditions and longer reaction times. Therefore, several modifications have been reported for the classical Hantzsch approach so far [10-23]. However, despite their potential efficacy, many of these methods still involve longer reaction time, two-step synthesis, expensive and toxic catalysts cumber, some harsh product isolation procedures and incompatibility with certain functional groups. Hence, the challenge for a sustainable environment calls for more general and viable routes that would be of great consequence to both synthetic and medicinal chemists is still demanded.<smiles>CCOC(=O)C1=C(COCCN)NC(C)=C(C(C)=O)C1c1ccccc1Cl</smiles>

Amlodipine<smiles>COC(=O)C1=C(C)NC(C)=C(C(=O)OCCN(C)Cc2ccccc2)C1c1cccc([N+](=O)[O-])c1</smiles>

Nicardipine<smiles>CCOC(=O)C1=C(C)NC(C)=C(C(C)=O)C1c1cccc(Cl)c1Cl</smiles>

Felodipine<smiles>COC(=O)C1=C(C)NC(C)=C(C(C)=O)C1c1ccccc1[N+](=O)[O-]</smiles>

Nifedipine

Figure 1. Some representative 1,4-dihdropyridines used as clinical drugs.

In addition, nanostructured materials due to large specific surface area and small diffusion resistance have received widespread attention, as a catalyst to synthesize useful heterocyclic compounds [24-29]. As most common nanostructures, metal oxides were applied due to their large surface area and high catalytic activities [30, 31]. In spite of some advantages, the tendency of metals nanoparticles for aggregation can have undesirable effects on their catalytic efficiency. The use of support to immobilize the metals nanoparticles can address this issue. Among the wide-ranging inorganic supports, mesoporous silica MCM-48 due to their outstanding structural characteristics like substantial pore volume, high stability, considerable surface area, uniform morphology of the pores, a simple modification of the surface and 3D interconnected pore network has been the center of major attention [32-36].

As a continuation of our ongoing endeavors in developing novel and practical multicomponent reactions to synthesize heterocyclic compounds of biological importance [37-45], we herein present the catalytic efficiency of $\mathrm{Ag} / \mathrm{CuO} / \mathrm{MCM}$ 48 for the multicomponent synthesis of symmetrical and unsymmetrical polyhydroquinoline derivatives under identical reaction conditions (Scheme 1). Recently, unsymmetrical polyhydroquinoline motifs have been recognized as lead molecules in antidiabetic drug discovery [46].

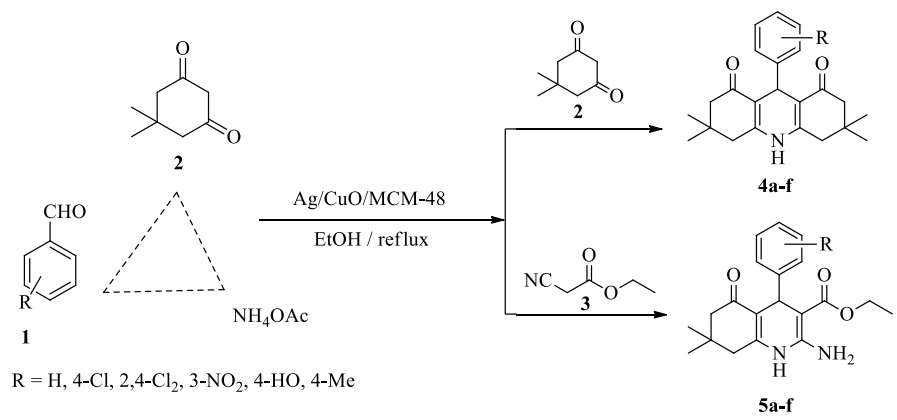

Scheme 1. Synthesis of symmetrical and unsymmetrical polyhydroquinolines.

\section{EXPERIMENTAL}

\subsection{Materials and apparatus}

All reagents and solvents were purchased from Merck and used without further modification. The ${ }^{1} \mathrm{H}$ NMR spectra were obtained on a Bruker Avance 400 and $500 \mathrm{MHz}$ spectrometers. The internal standard for the ${ }^{1} \mathrm{H}$ and ${ }^{13} \mathrm{C}$ NMR spectra 
was TMS. Chemical shifts $(\delta)$ are reported in ppm and the coupling constants $(J)$ are given in Hertz (Hz). The FT-IR spectra for the samples were obtained using Shimadzu FT-IR-8900 spectrophotometer by using KBr pellets. Field Emission Scanning Electron Microscopy (FE-SEM) was executed by a Hitachi S4160 instrument.

The low-angle XRD spectrum $\left(2 \theta=0.8-10^{\circ}\right)$ of $\mathrm{Ag} / \mathrm{CuO} / \mathrm{MCM}-48$ was subjected by X'Pert PRO MPD diffractometers with $\mathrm{Cu} \mathrm{K}_{\alpha}\left(\lambda=1.54056 \mathrm{~A}^{\circ}\right)$. Melting points were determined with an Electrothermal model 9100 apparatus and are uncorrected.

2.2. General procedure for the synthesis of symmetrical polyhydroquinoline (Table 2, 4a-f)

$\mathrm{Ag} / \mathrm{CuO} / \mathrm{MCM}-48$ was prepared according to our previous report [42]. Arylaldehyde 1 (1 mmol), dimedone 2 ( $2 \mathrm{mmol})$, ammonium acetate $(1.3 \mathrm{mmol})$ and $\mathrm{Ag} / \mathrm{CuO} / \mathrm{MCM}-48$ (30 mg) were taken in $4 \mathrm{~mL}$ EtOH in a round-bottomed flask and the reaction mixture was reflux for an appropriate reaction time (Table 2). After completion of the reaction which was monitored by TLC (eluent; $n$ hexane:EtOAc, 8:2), the reaction mixture was diluted with hot EtOH and $\mathrm{Ag} / \mathrm{CuO} / \mathrm{MCM}-48$ was separated by filtration. The filtrate was evaporated in a rotatory evaporator and the residue was recrystallized from EtOH to produce the pure products 4a-f in 83-95\% yield (Table 2). 2

2.3. General procedure for the synthesis of unsymmetrical polyhydroquinoline (Table 3 , 5a-f)

Arylaldehyde 1 ( $1 \mathrm{mmol})$, dimedone 2 (1 mmol), ethyl cyanoacetate $\mathbf{3}$ (1 mmol), ammonium acetate $(1.3 \mathrm{mmol})$ and $\mathrm{Ag} / \mathrm{CuO} / \mathrm{MCM}-48(30 \mathrm{mg})$ were mixed in $\mathrm{EtOH}(4 \mathrm{~mL})$ in a round-bottomed flask and heated at reflux condition. After completion of the reaction (monitored by TLC, eluent; n-hexane : EtOAc, $8: 2$ ), the reaction mixture was diluted with hot $\mathrm{EtOH}$ and the catalyst was separated by filtration. The filtrate was evaporated under vacuum and solid residue was recrystallized from EtOH to furnish the compounds 5a-f in 88-97\% yield (Table 4).

\section{Selected spectral data:}

2.4. 9-(4-Chlorophenyl)-3,3,6,6-tetramethyl-3,4,6,7,9,10-hexahydroacridine-1,8 $(2 H, 5 H)$-dione $4 a$

${ }^{1} \mathrm{H}$ NMR (500 MHz, DMSO-d $\left.{ }_{6}\right): \delta: 0.85\left(\mathrm{~s}, 6 \mathrm{H}, 2 \times \mathrm{CH}_{3}\right), 1.09\left(\mathrm{~s}, 6 \mathrm{H}, 2 \times \mathrm{CH}_{3}\right)$, $1.98(\mathrm{~d}, 2 \mathrm{H}, J=16.0 \mathrm{~Hz}, 2 \times \mathrm{CH}), 2.17(\mathrm{~d}, 2 \mathrm{H}, J=16.0 \mathrm{~Hz}, 2 \times \mathrm{CH}), 2.33(\mathrm{~d}, 2 \mathrm{H}$, $J=17.1 \mathrm{~Hz}, 2 \times \mathrm{CH}), 2.45(\mathrm{~d}, 2 \mathrm{H}, J=17.1 \mathrm{~Hz}, 2 \times \mathrm{CH}), 4.77(\mathrm{~s}, 1 \mathrm{H}, \mathrm{CH}), 7.15(\mathrm{~d}$, $2 \mathrm{H}, J=8.5 \mathrm{~Hz}, \mathrm{Ar}-\mathrm{H}), 7.22(\mathrm{~d}, 2 \mathrm{H}, J=8.5 \mathrm{~Hz}, \mathrm{Ar}-\mathrm{H}), 9.47(\mathrm{~s}, 1 \mathrm{H}, \mathrm{NH}) ;{ }^{13} \mathrm{CNMR}$

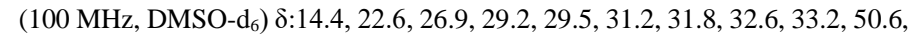
$70.3,111.5,128.0,129.9,130.4,146.6,150.0,194.9,207.0 \mathrm{ppm}$.

2.5. Ethyl 2-amino-4-(4-chlorophenyl)-7,7-dimethyl-5-oxo-1,4,5,6,7,8hexahydroquinoline-3-carboxylate 5a

${ }^{1} \mathrm{H}$ NMR (500 MHz, DMSO-d 6 ) $\delta: 0.83\left(\mathrm{~s}, 3 \mathrm{H}, \mathrm{CH}_{3}\right), 1.00\left(\mathrm{~s}, 3 \mathrm{H}, \mathrm{CH}_{3}\right), 1.08$ $\left(\mathrm{t}, 3 \mathrm{H}, J=7.1 \mathrm{~Hz}, \mathrm{CH}_{3}\right), 1.93(\mathrm{~d}, 1 \mathrm{H}, J=16.0 \mathrm{~Hz}, \mathrm{CH}), 2.15(\mathrm{~d}, 1 \mathrm{H}, J=16.0 \mathrm{~Hz}$, $\mathrm{CH}), 2.27(\mathrm{~d}, 1 \mathrm{H}, J=17.1 \mathrm{~Hz}, \mathrm{CH}), 2.40(\mathrm{~d}, 1 \mathrm{H}, J=17.1 \mathrm{~Hz}, \mathrm{CH}), 3.86-3.92(\mathrm{~m}$, $2 \mathrm{H}, \mathrm{CH}_{2}$ ), 4.68 (s, 1H, CH), 7.13 (d, 2H, $\left.J=8.5 \mathrm{~Hz}, \mathrm{ArH}\right), 7.20$ (d, 2H, $J=8.5$ $\mathrm{Hz}, \mathrm{ArH}) \mathrm{ppm}$.

\section{RESULTS AND DISCUSSION}

Following our prior efforts to prepare heterogeneous catalysts and apply them for the synthesis of heterocyclic systems [38, 40-42, 44, 45], herein, a facile Hantzsch condensation in the presence of $\mathrm{Ag} / \mathrm{CuO} / \mathrm{MCM}-48$ as an effective heterogeneous and reusable catalyst in $\mathrm{EtOH}$ has been employed to obtain diversely substituted symmetrical and unsymmetrical polyhydroquinoline 4a-f and 5a-f with high to excellent yields (Scheme 1). Ag/CuO/MCM-48 was synthesized by the procedure described in our previous report [42].

The structure of prepared catalyst was identified by IR, XRD and SEM. In the FT-IR spectrum of Ag/CuO/MCM-48, the O-H stretching and bending modes in silanols and adsorbed water is observed at 3441 and $1638 \mathrm{~cm}^{-1}$. The appeared bands at 1103,805 and $469 \mathrm{~cm}^{-1}$ can be corresponded to the asymmetric, symmetric and bending vibrations of Si-O-Si bridges, respectively (Fig. 2).

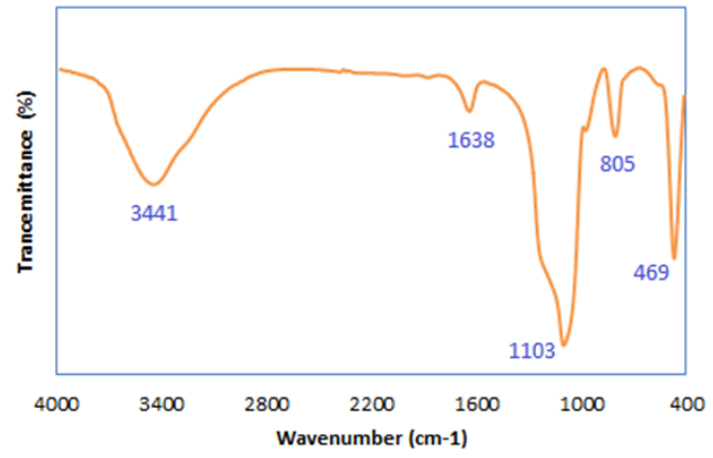

Figure 2. FT-IR spectrum of $\mathrm{Ag} / \mathrm{CuO} / \mathrm{MCM}-48$.

The spherical morphology of Ag/CuO/MCM-48 was confirmed by the FESEM image (Fig. 3). The size of the particles is nearly $200-900 \mathrm{~nm}$.

The low angle XRD diffraction spectrum of $\mathrm{Ag} / \mathrm{CuO} / \mathrm{MCM}-48$ shows the peak correlated to Bragg plane (211) of Ia3d cubic MCM-48 at around $2 \theta=2.3^{\circ}[42$, 47] (Fig. 4).

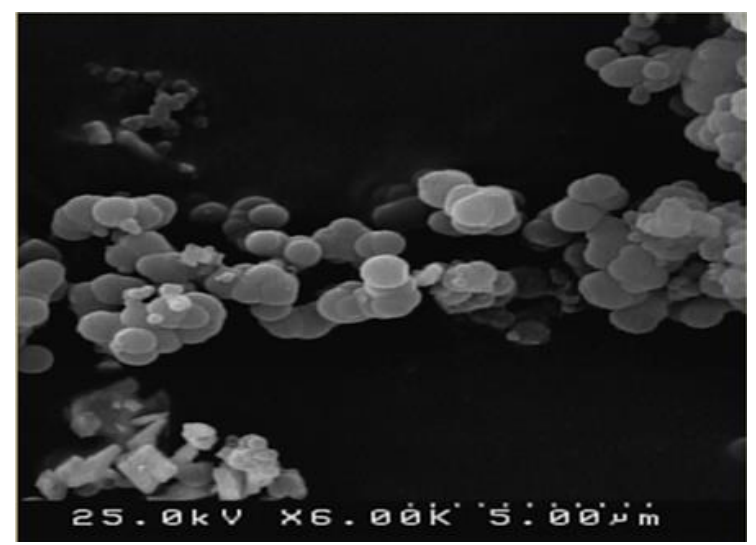

Figure 3. SEM image of $\mathrm{Ag} / \mathrm{CuO} / \mathrm{MCM}-48$

After preparation of the catalyst an initial screen of reaction conditions was carried out, therefore a mixture of 4-chlorobenzaldehyde $1 \mathbf{a}(1 \mathrm{mmol})$, dimedone $2(2 \mathrm{mmol})$ and ammonium acetate $(1.3 \mathrm{mmol})$ were stirred for $360 \mathrm{~min}$ at room temperature in $\mathrm{EtOH}$. This reacrion produced polyhydroquinoline $\mathbf{4 a}$ in $40 \%$ yield (Table 1, entry 1). Further verification of the effect of the amount of catalyst, solvent and temperature (Table 1, entries 2-9) revealed that using $30 \mathrm{mg}$ $\mathrm{Ag} / \mathrm{CuO} / \mathrm{MCM}-48$ in $\mathrm{EtOH}$ at reflux condition produces the product $4 \mathbf{a}$ in $95 \%$ yield (Table 1, entry 2).

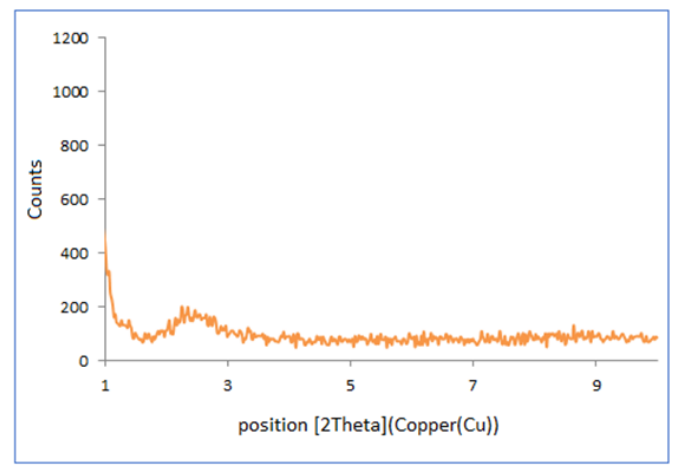

Figure 4. The low angle X-ray diffraction patterns of Ag/CuO/MCM-48.

The protocol was applied for the synthesis of symmetrical polyhydroquinoline (4a-f). Various arylaldehydes having electron-donating as well as electronwithdrawing substituents were used for the synthesis of corresponding polyhydroquinolines under the optimized reaction conditions in high to excellent yields (83-95\%) (Table 2). 
Table1. Optimization of the synthesis of $\mathbf{4 a}$ under various conditions.

\begin{tabular}{|c|c|c|c|c|c|c|}
\hline Entry & Catalyst & Amount of the catalyst (mg) & Solvent & Temp. $\left({ }^{\circ} \mathrm{C}\right)$ & $\begin{array}{l}\text { Time } \\
(\min )\end{array}$ & Yield $^{\mathrm{a}}(\%)$ \\
\hline 1 & - & - & $\mathrm{EtOH}$ & reflux & 360 & 40 \\
\hline 2 & $\mathrm{Ag} / \mathrm{CuO} / \mathrm{MCM}-48$ & 30 & EtOH & reflux & 20 & 95 \\
\hline 3 & $\mathrm{Ag} / \mathrm{CuO} / \mathrm{MCM}-48$ & 20 & $\mathrm{EtOH}$ & reflux & 25 & 81 \\
\hline 4 & $\mathrm{Ag} / \mathrm{CuO} / \mathrm{MCM}-48$ & 40 & $\mathrm{EtOH}$ & reflux & 20 & 95 \\
\hline 5 & $\mathrm{Ag} / \mathrm{CuO} / \mathrm{MCM}-48$ & 30 & $\mathrm{EtOH}$ & r.t. & 80 & 70 \\
\hline 6 & $\mathrm{Ag} / \mathrm{CuO} / \mathrm{MCM}-48$ & 30 & DMF & reflux & 180 & 68 \\
\hline 7 & $\mathrm{Ag} / \mathrm{CuO} / \mathrm{MCM}-48$ & 30 & $\mathrm{H}_{2} \mathrm{O}$ & reflux & 200 & 70 \\
\hline 8 & $\mathrm{Ag} / \mathrm{CuO} / \mathrm{MCM}-48$ & 30 & $\mathrm{CH}_{3} \mathrm{CN}$ & reflux & 180 & 73 \\
\hline 9 & $\mathrm{Ag} / \mathrm{CuO} / \mathrm{MCM}-48$ & 30 & $\mathrm{MeOH}$ & reflux & 25 & 84 \\
\hline
\end{tabular}

${ }^{\mathrm{a}}$ Isolated Yield.

Table 2. $\mathrm{Ag} / \mathrm{CuO} / \mathrm{MCM}-48$ catalyzed synthesis of symmetrical polyhydroquinoline $\mathbf{4 a - f}$.

\begin{tabular}{|c|c|c|c|c|c|c|c|c|}
\hline \multirow{2}{*}{ Entry } & \multirow{2}{*}{ Aldehyde } & & \multirow{2}{*}{ Product } & \multirow{2}{*}{ Time (min) } & \multirow{2}{*}{$\begin{array}{l}\text { Yield } \\
(\%)^{\mathrm{a}}\end{array}$} & \multirow[b]{2}{*}{ Found } & \multicolumn{2}{|c|}{$\mathbf{M p}{ }^{\circ} \mathbf{C}$} \\
\hline & & & & & & & Reported & Ref. \\
\hline 1 & $4-\mathrm{ClC}_{6} \mathrm{H}_{4} \mathrm{CHO}$ & $4 \mathrm{a}$ & & 20 & 95 & $>300$ & $317-320$ & [48] \\
\hline 2 & $3-\mathrm{O}_{2} \mathrm{NC}_{6} \mathrm{H}_{4} \mathrm{CHO}$ & $4 b$ & & 25 & 88 & $293-295$ & 296-297 & [49] \\
\hline 3 & 2,4- $\mathrm{Cl}_{2} \mathrm{C}_{6} \mathrm{H}_{3} \mathrm{CHO}$ & $4 \mathrm{c}$ & & 20 & 90 & $>300$ & - & \\
\hline 4 & 4- $\mathrm{HOC}_{6} \mathrm{H}_{4} \mathrm{CHO}$ & $4 d$ & & 35 & 86 & $282-284$ & 284-286 & [48] \\
\hline 5 & 4- $\mathrm{MeC}_{6} \mathrm{H}_{4} \mathrm{CHO}$ & $4 \mathrm{e}$ & & 35 & 85 & $282-284$ & $279-281$ & [48] \\
\hline 6 & $\mathrm{C}_{6} \mathrm{H}_{5} \mathrm{CHO}$ & $4 \mathrm{f}$ & & 35 & 83 & $277-279$ & $277-279$ & [48] \\
\hline
\end{tabular}


Table3. Optimization of the reaction conditions for the synthesis of $\mathbf{5 a}$.

\begin{tabular}{|c|c|c|c|c|c|c|}
\hline Entry & Catalyst & Amount of the catalyst (mg) & Solvent & Temp. $\left({ }^{\circ} \mathrm{C}\right)$ & $\begin{array}{l}\text { Time } \\
(\mathrm{min})\end{array}$ & Yield $^{\mathrm{a}}(\%)$ \\
\hline 1 & - & & $\mathrm{EtOH}$ & reflux & 360 & 25 \\
\hline 2 & $\mathrm{Ag} / \mathrm{CuO} / \mathrm{MCM}-48$ & 30 & EtOH & reflux & 18 & 97 \\
\hline 3 & $\mathrm{Ag} / \mathrm{CuO} / \mathrm{MCM}-48$ & 20 & EtOH & reflux & 25 & 88 \\
\hline 4 & $\mathrm{Ag} / \mathrm{CuO} / \mathrm{MCM}-48$ & 40 & $\mathrm{EtOH}$ & reflux & 18 & 96 \\
\hline 5 & $\mathrm{Ag} / \mathrm{CuO} / \mathrm{MCM}-48$ & 30 & $\mathrm{EtOH}$ & r.t. & 60 & 74 \\
\hline 6 & $\mathrm{Ag} / \mathrm{CuO} / \mathrm{MCM}-48$ & 30 & DMF & reflux & 140 & 72 \\
\hline 7 & $\mathrm{Ag} / \mathrm{CuO} / \mathrm{MCM}-48$ & 30 & $\mathrm{H}_{2} \mathrm{O}$ & reflux & 180 & 74 \\
\hline 8 & $\mathrm{Ag} / \mathrm{CuO} / \mathrm{MCM}-48$ & 30 & $\mathrm{CH}_{3} \mathrm{CN}$ & reflux & 150 & 77 \\
\hline 9 & $\mathrm{Ag} / \mathrm{CuO} / \mathrm{MCM}-48$ & 30 & $\mathrm{MeOH}$ & reflux & 20 & 87 \\
\hline
\end{tabular}

${ }^{\mathrm{a}}$ Isolated Yield.

Table 4. $\mathrm{Ag} / \mathrm{CuO} / \mathrm{MCM}-48$ catalyzed synthesis of unsymmetrical polyhydroquinoline 5a-f.

\begin{tabular}{|c|c|c|c|c|c|c|c|c|}
\hline \multirow{2}{*}{ Entry } & \multirow{2}{*}{ Aldehyde } & & \multirow{2}{*}{ Product } & \multirow{2}{*}{ Time (min) } & \multirow{2}{*}{$\begin{array}{l}\text { Yield } \\
(\%)^{\mathrm{a}}\end{array}$} & \multirow[b]{2}{*}{ Found } & \multicolumn{2}{|c|}{$\mathbf{M p}{ }^{\circ} \mathbf{C}$} \\
\hline & & & & & & & Reported & Ref. \\
\hline 1 & 4- $\mathrm{ClC}_{6} \mathrm{H}_{4} \mathrm{CHO}$ & $5 \mathrm{a}$ & & 18 & 97 & $154-156$ & $155-157$ & {$[50]$} \\
\hline 2 & $3-\mathrm{O}_{2} \mathrm{NC}_{6} \mathrm{H}_{4} \mathrm{CHO}$ & $5 b$ & & 25 & 85 & $185-187$ & $183-184$ & {$[50]$} \\
\hline 3 & $2,4-\mathrm{Cl}_{2} \mathrm{C}_{6} \mathrm{H}_{3} \mathrm{CHO}$ & $5 c$ & & 17 & 95 & $182-184$ & - & \\
\hline 4 & 4- $\mathrm{HOC}_{6} \mathrm{H}_{4} \mathrm{CHO}$ & $5 d$ & & 30 & 91 & $148-150$ & - & \\
\hline 5 & $4-\mathrm{MeC}_{6} \mathrm{H}_{4} \mathrm{CHO}$ & $5 \mathrm{e}$ & & 30 & 89 & $141-143$ & $139-141$ & {$[50]$} \\
\hline 6 & $\mathrm{C}_{6} \mathrm{H}_{5} \mathrm{CHO}$ & $5 \mathrm{f}$ & & 30 & 88 & $154-156$ & $157-160$ & {$[50]$} \\
\hline
\end{tabular}

\section{${ }^{a}$ Isolated Yield}

A possible mechanism for the synthesis of unsymmetrical polyhydroquinolines catalyzed by $\mathrm{Ag} / \mathrm{CuO} / \mathrm{MCM}-48$ is demonstrated in Scheme 3. The Lewis acid nature of the catalyst can activate the reactants. First, Knoevenagel adduct $\mathbf{A}$ is formed by the reaction of arylaldehyde and ethyl cyanoacetate (3). Simultaneously, the reaction of ammonium acetate and dimedone (2) leads to intermediate $\mathbf{B}$. Then, intermediate $\mathbf{C}$ is produced by Michael addition of intermediate $\mathbf{B}$ on arylidene $\mathbf{A}$. Eventually, the intramolecular cyclization afforded the desired polyhydroquinoline derivatives (5a-f).

From an economical point of view, the recyclability of $\mathrm{Ag} / \mathrm{CuO} / \mathrm{MCM}-48$ was also considered. To achieve this purpose, after completion of the synthesis of
9-(4-chlorophenyl)-3,3,6,6-tetramethyl-3,4,6,7,9,10-hexahydroacridine-1,8(2H,5H)dione 4a and ethyl 2-amino-4-(4-chlorophenyl)-7,7-dimethyl-5-oxo-1,4,5,6,7,8hexahydroquinoline-3-carboxylate 5a the hot ethanol was added to the reaction mixture. After the filtration, the separated catalyst washed with hot ethanol and dried at $100{ }^{\circ} \mathrm{C}$. Then, it was reused for the subsequent cycle. $\mathrm{Ag} / \mathrm{CuO} / \mathrm{MCM}-48$ retained high reactivity for up to 10 cycles (Fig 5,6).

The efficiency of the catalyst to promote the synthesis of 9-(4-chlorophenyl)3,3,6,6-tetramethyl-3,4,6,7,9,10-hexahydroacridine-1,8(2H,5H)-dione $\mathbf{4 a}$ and ethyl 4-(4-chlorophenyl)-2,7,7-trimethyl-5-oxo-1,4,5,6,7,8-hexahydroquinoline3 -carboxylate 5a was compared with some reported catalysts. As illustrated in Table 5, high capability of the present catalyst was obviously observed. 


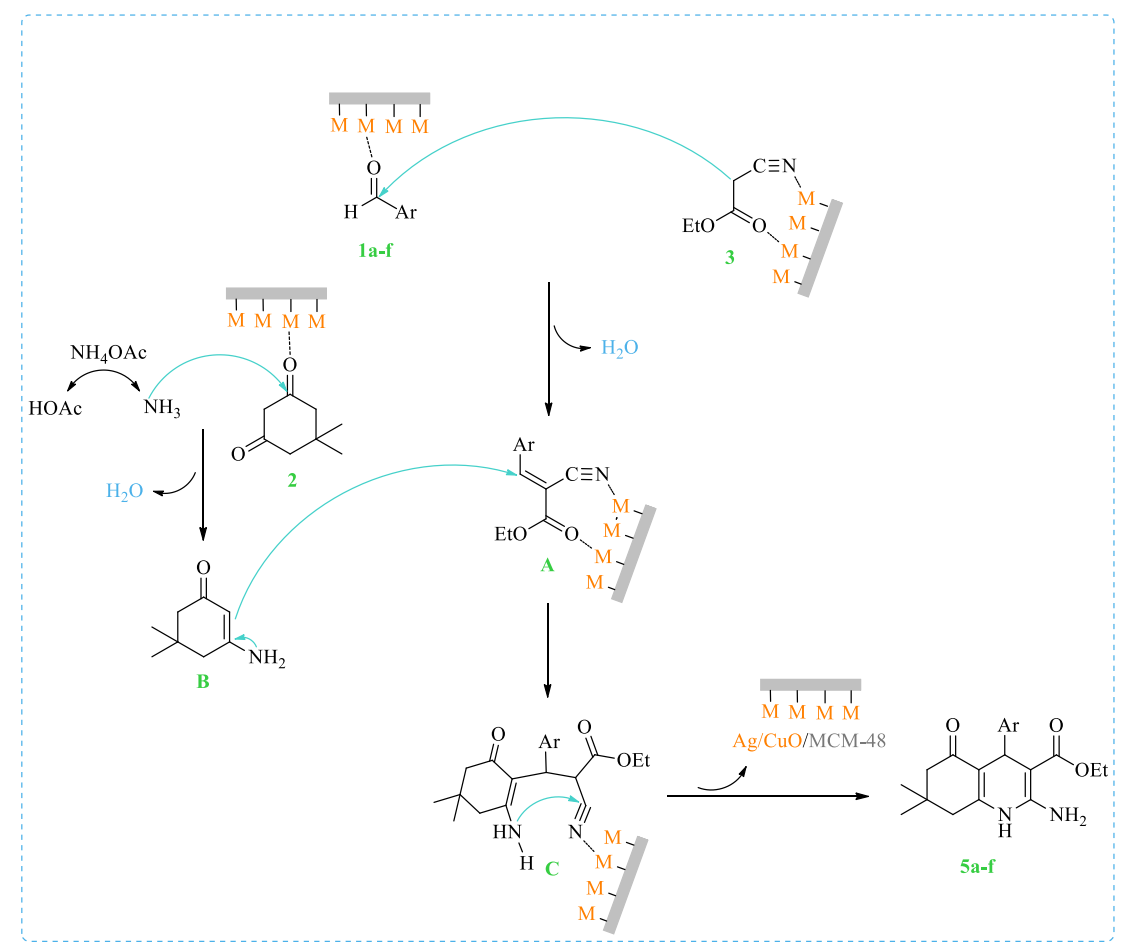

Scheme 2. A plausible mechanism for the synthesis of 5a-f in the presence of Ag/CuO/MCM-48.

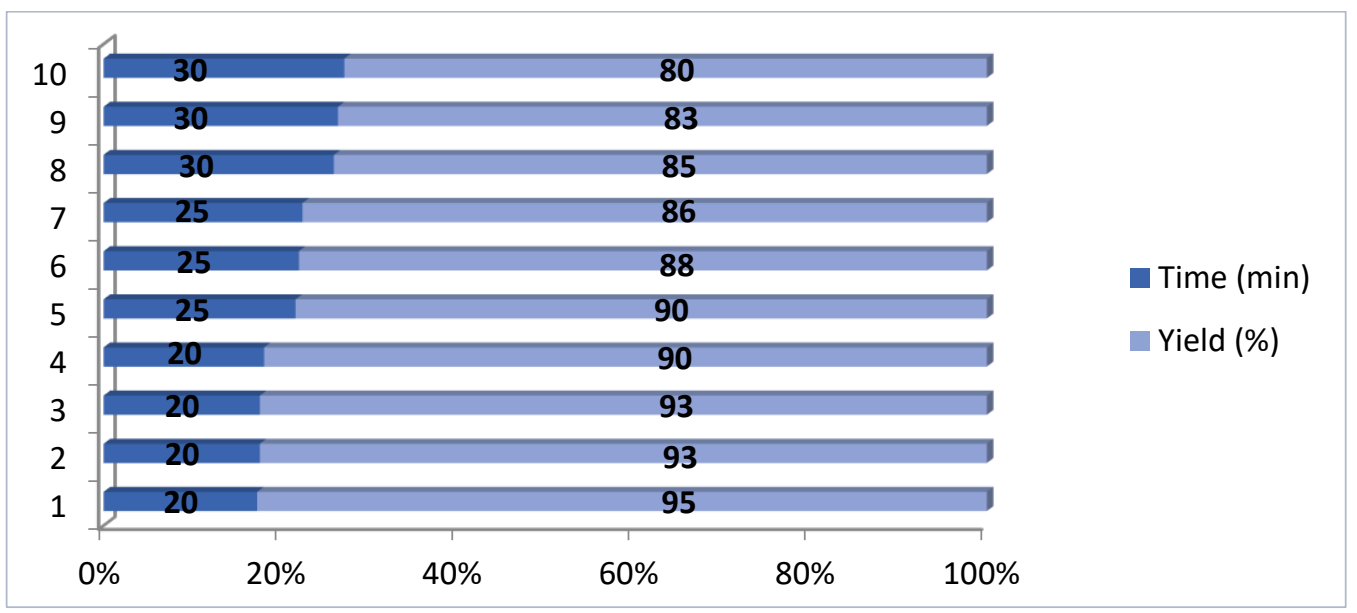

Figure 5. Recyclability of the catalyst $\mathrm{Ag} / \mathrm{CuO} / \mathrm{MCM}-48$ in the synthesis of $\mathbf{4 a}$.

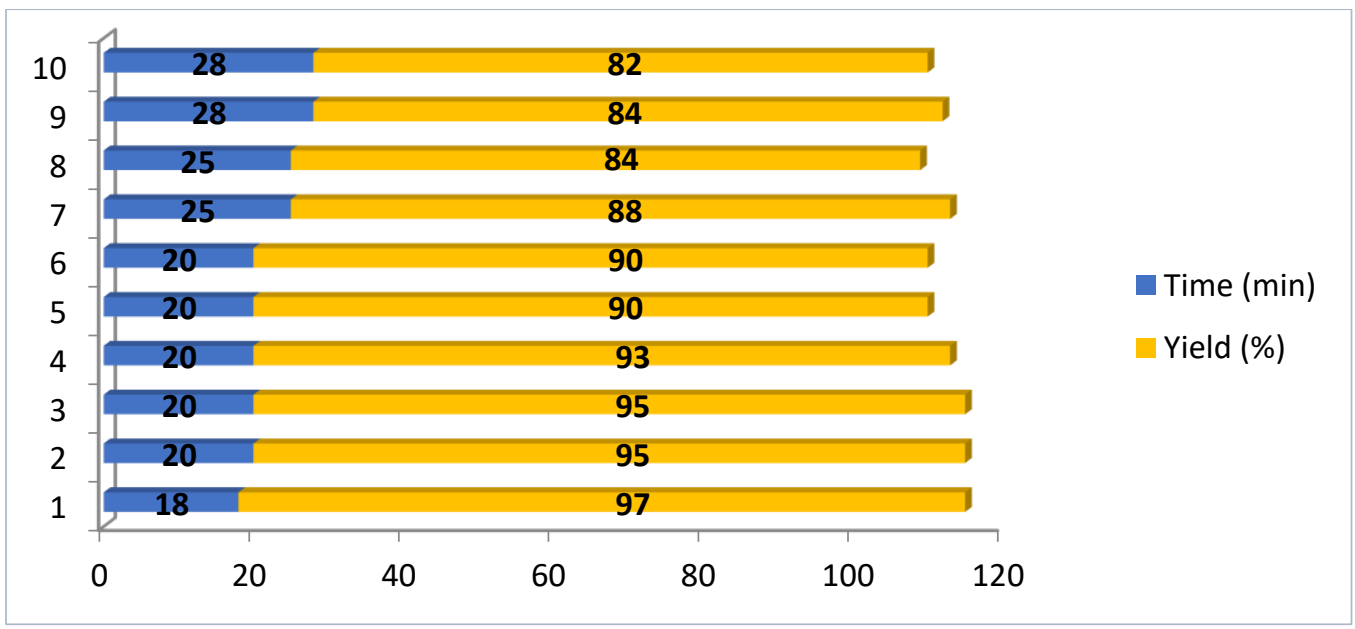

Figure 6. Recyclability of the catalyst $\mathrm{Ag} / \mathrm{CuO} / \mathrm{MCM}-48$ in the synthesis of $\mathbf{5 a}$. 
Table 5. Comparison of catalytic effect of $\mathrm{Ag} / \mathrm{CuO} / \mathrm{MCM}-48$ with other reported catalysts for the preparation of $\mathbf{4 a}$ and $\mathbf{5 a}$.

\begin{tabular}{|c|c|c|c|c|c|c|c|}
\hline Entry & Product & Catalyst & Amount & Condition & Time (min) & $\begin{array}{l}\text { Yield } \\
(\%)\end{array}$ & Ref. \\
\hline 1 & & $\mathrm{SiO}_{2}-\mathrm{I}$ & $0.25 \mathrm{~g}$ & $\mathrm{EtOH} / 80^{\circ} \mathrm{C}$ & 90 & 82 & [51] \\
\hline 2 & & $\mathrm{SiO}_{2}-\mathrm{Pr}-\mathrm{SO}_{3} \mathrm{H}$ & $0.02 \mathrm{~g}$ & Solvent-free $/ 120^{\circ} \mathrm{C}$ & 120 & 95 & [48] \\
\hline 3 & & $\mathrm{Ag} / \mathrm{CuO} / \mathrm{MCM}-48$ & $0.03 \mathrm{~g}$ & EtOH/reflux & 20 & 95 & This work \\
\hline 4 & & Pd NPs & $\begin{array}{c}0.04 \\
\mathrm{mmol}\end{array}$ & $\mathrm{THF} /$ reflux & 240 & 87 & [52] \\
\hline 5 & & $\mathrm{Fe}_{3} \mathrm{O}_{4} @ \mathrm{~B}-\mathrm{MCM}-41$ & $0.05 \mathrm{~g}$ & EtOH/reflux & 40 & 92 & [53] \\
\hline 6 & & FSM-16- $\mathrm{SO}_{3} \mathrm{H}$ & $0.04 \mathrm{~g}$ & EtOH/reflux & 20 & 88 & {$[50]$} \\
\hline 7 & $\begin{array}{r}\mathrm{N} \\
\mathrm{H}\end{array}$ & $\mathrm{Ag} / \mathrm{CuO} / \mathrm{MCM}-48$ & $0.03 \mathrm{~g}$ & EtOH/reflux & 18 & 97 & This work \\
\hline
\end{tabular}

\section{CONCLUSION}

In summary, a simple and highly efficient one-pot multi-component reaction for the synthesis of symmetrical and unsymmetrical polyhydroquinoline derivatives using $\mathrm{Ag} / \mathrm{CuO} / \mathrm{MCM}-48$ catalyst was developed. This methodology benefits from high to excellent yield of the desired products, use of eco-friendly solvent, no requiring laborious work-up, simple purification of the product without using chromatographic procedures and ability to recycle the catalyst.

\section{ACKNOWLEDGMENTS}

The authors are grateful to the Research Council of University of Guilan for the partial financial support.

\section{SUPPLEMENTARY DATA}

The ${ }^{1} \mathrm{H}$ NMR and ${ }^{13} \mathrm{C}$ NMR spectra of compounds are available in supporting information.

\section{REFERENCES}

1. F. Bossert, H. Meyer, E. Wehinger, Angew. Chem., Int. Ed. Engl. 20, 762, (1981)

2. H. Nakayama, Y. Kasoaka, Heterocycles 42, 901, (1996)

3. T. Godfraid, R. Miller, M. Wibo, Pharmocol. Rev. 38, 321, (1986)

4. R. G. Bretzel, C. C. Bollen, E. Maester, K. F. Federlin, Drugs Future 17, 465, (1992)

5. P. N. Kalaria, S. P. Satasia, D. K. Raval, Eur. J. Med. Chem. 78, 207, (2014)

6. 6.- G. C. Rovnyak, S. D. Kimball, B. Beyer, G. Cucinotta, J. D. Dimarco, J. Gougoutas, A. Hedberg, M. Malley, J. P. MaCarthy, R. Zhang, S. Mereland, J. Med. Chem. 38, 119, (1995)

7. C. O. Kappe, W. M. F. Fabian, M. A. Semones, Tetrahedron 53, 2803, (1997)

8. K. Aouam, A. Berdeaux, Therapie 58, 333, (2003)

9. A. Hilgeroth, Mini-Rev. Med. Chem. 2, 235, (2002)

10. U. Eisner, J. Kuthan, Chem. Rev. 72, 1, (1972)

11. D. M. Stout, A. I. Meyers, Chem. Rev. 82, 223, (1982)

12. A. Sausins, G. Duburs, Heterocycles 27, 269, (1988)

13. N. Koukabi, E. Kolvari, A. Khazaei, M. A. Zolfigol, B. S. Shaghasemi, H. R. Khavasi, Chem. Commun. 47, 9230, (2011)

14. R. Ananthakrishnan, S.Gazi, Catal. Sci. Technol. 2, 1463, (2012)

15. L. Shen, S. Cao, J. Wu, J. Zhang, H. Li, N. Liu, X. Qian, Green Chem. 11, 1414, (2009)

16. M. M. Aghayan, R. Boukherroub, M. Nemati, M. Rahimifard, Tetrahedron Lett. 53, 2473, (2012)

17. A. Debache, R. Boulcina, A. Belfaitah, S. Rhouti, B.Carboni, Synlett 4, 509, (2008)

18. S. Thakrar, A. Bavishi, D. Bhavsar, S. Parekh, H. Vala, A. Radadiya, M. Parmar, M. Savant, Synth. Commun. 42, 3269, (2012)

19. S. Rostamnia, K. Lamei, Chin. Chem. Lett. 23, 930, (2012)

20. A. Kuraitheerthakumaran, S. Pazhamalaian, M. Gopalakrishnan, Chin. Chem. Lett. 22, 1199 (2011)

21. N. Koukabi, E. Kolvari, M. A. Zolfigol, A. Khazaei, B. S. Shaghasemi, B. Fasahati, Adv. Synth. Catal., 354, 2001, (2012)
22. R. Kumar, N. H. Andhare, A. Shard, A. Richa, K. Sinha. RSC. Adv. 4, 19111 (2014)

23. D. Langle, V. Marquardt, E. Heider, B. Vigante, G. Duburs, I. Luntena, D. Flotgen, C. Golz, C. Strohmann, Oliver Koch, D. Schade, Eur. J. Med. Chem. 95, 249, (2015)

24. F. Zaera, Chem. Soc. Rev. 42, 2746, (2013)

25. N. RaveendranShijuVadim V.Guliants, Appl. Catal., A 356,1, (2009)

26. A. H. Lu, E. L. Salabas, F. Schuth, Angew. Chem. Int. Ed. 46, 1222, (2007)

27. X. Wu, Y. Guo, L. Wan, C. Hu, J. Phys. Chem. 112, 16824, (2008)

28. A. G. Nasibulin, S. Rackauskas, H. Jiang, Nanopart. Res. 2, 373, (2009)

29. Z. Guo, D. Zhang, S. Wei, Z. Wang, B. Karki, J. Nanopart. Res. 12, 2415, (2010)

30. S. B. Sapkal,K. F. Shelke, B. B. Shingate, M. S. Shingare, Tetrahedron Lett. 50, 1754, (2009)

31. M. Abdollahi-Alibeik, S-S. Hoseinikhah, J. Iran Chem. Soc. 13, 1339, (2016)

32. R. J. Kalbasi, N. Mosaddegh, Catal. Commun. 12, 1231, (2011)

33. E. Vessally, R. Hosseinzadeh-Khanmiri, E. Ghorbani-Kalhor, M. Eshaghi, L. Ejlali, Appl. Organomet. Chem. 31, e3729, (2017)

34. W. Zhan, Y. Guo, Y. Wang, Y. Guo, X. Liu, Y. Wang, Z. Zhang, G. Lu, J. Phys. Chem. C 113, 7181, (2009)

35. N. Mosaddegh, I. Yavari, Chem. Pap. 72, 2013, (2018)

36. Y. Duan, D. Zhai, X. Zhang, J. Zheng, C. Li, Catal. Lett. 148, 51, (2018)

37. L. Kheirkhah, M. Mamaghani, A. Yahyazadeh, N. O. Mahmoodi, Appl. Organomet. Chem. 32, e4072, (2018)

38. M. Ahmadiazar, M. Mamaghani, Curr. Org. Chem. 22, 1326, (2018)

39. F. Tavakoli, M. Mamaghani, M. Sheykhan, N. Mohammadipour, M. Rassa, Curr. Org. Synth. 15, 872, (2018)

40. M. Mamaghani, M. Sheykhan, M. Sadeghpour, F. Tavakoli, Monatsh. Chem. 149, 1437, (2018)

41. P. Jahanshahi, M. Mamaghani, F. Haghbin, R. Hossein Nia, M. Rassa, J. Mol. Struct. 1155, 520, (2018)

42. F. Tavakoli, M. Mamaghani, M. Sheykhan, Appl. Organomet. Chem. 33, e5083, (2019)

43. P. Jahanshahi, M. Mamaghani, New J. Chem. 43, 8266, (2019)

44. F. Ramezanzadeh, M. Mamaghani, H. Fallah-Bagher Shaidaei, M. Sheykhan, Polycycl. Aromat. Comp. DOI: 10.1080/10406638.2019.1705360, (2019)

45. E. Saberikhah, M. Mamaghani, N. O. Mahmoodi,; A. Fallah Shojaei, Polycycl. Aromat. Comp. DOI: 10.1080/10406638.2020.1729821, (2020)

46. A. Kumar, S. Sharma, V. D. Tripathi, R. A. Maurya, S. P. Srivastava, G. Bhatia, A. K. Tamrakar, A. K. Srivastava, Bioorg. Med. Chem. 18, 4138, (2010)

47. C. Witpathomwong, R. Longloilert, S. Wongkasemjit, S. Jitkarnka, Energy Procedia 9, 245, (2011)

48. G. M.Ziarani, A. Badiei, M. Hassanzadeh, S. Mousavi, Arab. J. Chem. 7, 335, (2014)

49. G.-W. Wang, J.-J.Xia, C.-B. Miao, X.-L. Wu, Bull. Chem. Soc. Jpn. 79, 454, (2006)

50. S. Hashemi-Uderji, M. Abdollahi-Alibeik, R. Ranjbar-Karimi, Main Group Met. Chem. 41, 91, (2018)

51. K. Ramesh, M. Pasha, Bioorg. Med. Chem. Lett. 24, 3907, (2014)

52. M. Saha, A. K. Pal, Tetrahedron Lett. 52, 4872, (2011)

53. M. Abdollahi-Alibeik, A. Rezaeipoor-Anari, J. Magn. Magn. Mater. 398, 205, (2016) 